\title{
Exact Solutions to the Boussinesq-Burgers Equations
}

\author{
Xiangzheng Li ${ }^{*}$, Baoan Li1, Jinlan Chen', Mingliang Wang ${ }^{1,2}$ \\ ${ }^{1}$ School of Mathematics and Statistics, Henan University of Science and Technology, Luoyang, China \\ ${ }^{2}$ School of Mathematics and Statistics, Lanzhou University, Lanzhou, China \\ Email: *xiangzheng_li@qq.com
}

How to cite this paper: Li, X.Z., Li, B.A., Chen, J.L. and Wang, M.L. (2017) Exact Solutions to the Boussinesq-Burgers Equations. Journal of Applied Mathematics and Physics, 5, 1720-1724.

https://doi.org/10.4236/jamp.2017.59145

Received: August 15, 2017

Accepted: September 15, 2017

Published: September 18, 2017

Copyright (C) 2017 by authors and Scientific Research Publishing Inc. This work is licensed under the Creative Commons Attribution International License (CC BY 4.0).

http://creativecommons.org/licenses/by/4.0/

\begin{abstract}
A nonlinear transformation from the solution of a linear equation to the solution of the Boussinesq-Burgers equations is derived by using the simplified homogeneous balance method. Based on the nonlinear transformation and various given solutions of the linear equation, various exact solutions, including solitary wave solutions, rational solutions, the solutions containing hyperbolic functions and the solutions containing trigonometric functions, of the Boussinesq-Burgers equations are obtained.
\end{abstract}

\section{Keywords}

Boussinesq-Burgers Equations, Nonlinear Transformation, Simplified Homogeneous Balance Method, Exact Solutions

\section{Introduction}

In the present paper, we will investigate the well-known Boussinesq-Burgers equations in the form

$$
\begin{gathered}
u_{t}+2 u u_{x}-\alpha v_{x}=0, \\
v_{t}+2(u v)_{x}-\alpha u_{x x x}=0,
\end{gathered}
$$

where $\alpha=$ constant. Equations ((1) \& (2)) emerge in the investigation of fluid flow, and describe the proliferation of shallow water waves. $u=u(x, t)$ represents horizontal velocity; $v=v(x, t)$ represents the height of the water surface above horizontal at bottom. Equations ((1) \& (2)) have been investigated by many authors with different methods (see [1]-[6] and references therein).

In this paper, we will propose a somewhat different method to find various exact solutions of Equations ((1) \& (2)). First of all, a nonlinear transformation from the solution of a linear equation to the solution of Equations ((1) \& (2)) is 
derived by using the simplified homogeneous balance method [7] [8] [9]; then based on the nonlinear transformation, various exact solutions of Equations ((1) $\&(2))$ can be obtained by the given various solutions of the linear equation.

\section{Derivation of Nonlinear Transformation}

Considering the homogeneous balance between $u u_{x}$ and $v_{x}$ in Equation (1), and between $(u v)_{x}$ and $u_{x x x}$ in Equation (2):

$$
2 m+1=n+1, m+n+1=m+3 \Rightarrow m=1, n=2,
$$

according to the simplified homogeneous balance method [7] [8] [9], we can suppose that the solution of Equations ((1) \& (2)) is of the form

$$
\begin{aligned}
& u(x, t)=A(\ln \phi)_{x}, \\
& v(x, t)=B(\ln \phi)_{x x},
\end{aligned}
$$

where the constants $A$ and $B$, as well as the function $\phi=\phi(x, t)$ are to be determined later. Substituting (3), (4) into the left hand sides of Equations ((1) \& (2)), yields

$$
\begin{aligned}
& u_{t}+2 u u_{x}-\alpha v_{x}=A \frac{\partial}{\partial x}\left[\frac{\phi_{t}-\alpha \frac{B}{A} \phi_{x x}}{\phi}+\left(A+\alpha \frac{B}{A}\right)\left(\frac{\phi_{x}}{\phi}\right)^{2}\right], \\
& v_{t}+2(u v)_{x}-\alpha u_{x x x}=B \frac{\partial^{2}}{\partial_{x}^{2}}\left[\frac{\phi_{t}-\alpha \frac{A}{B} \phi_{x x}}{\phi}+\left(A+\alpha \frac{A}{B}\right)\left(\frac{\phi_{x}}{\phi}\right)^{2}\right] .
\end{aligned}
$$

In expressions (5), (6), setting the coefficients of $\left(\frac{\phi_{x}}{\phi}\right)^{2}$ to zero, yields

$$
A+\alpha \frac{B}{A}=0, A+\alpha \frac{A}{B}=0 \Rightarrow A= \pm \alpha, B=-\alpha .
$$

Using the results (7), the expressions (3), (4) becomes

$$
\begin{aligned}
& u(x, t)= \pm \alpha(\ln \phi)_{x}, \\
& v(x, t)=-\alpha(\ln \phi)_{x x},
\end{aligned}
$$

and the expressions (5), (6) can be simplified as

$$
\begin{aligned}
& u_{t}+2 u u_{x}-\alpha v_{x}= \pm \alpha \frac{\partial}{\partial x}\left[\frac{\phi_{t} \pm \alpha \phi_{x x}}{\phi}\right]=0, \\
& v_{t}+2(u v)_{x}-\alpha u_{x x x}=-\alpha \frac{\partial^{2}}{\partial_{x}^{2}}\left[\frac{\phi_{t} \pm \alpha \phi_{x x}}{\phi}\right]=0,
\end{aligned}
$$

provided that the function $\phi=\phi(x, t)$ satisfies the linear equation

$$
\phi_{t} \pm \alpha \phi_{x x}=0 \text {. }
$$

From (8)-(12), we come to the conclusion that if $\phi=\phi(x, t)$ is a solution of 
the Equation (12), substituting it into the expressions (8), (9), we have the solution of Equations ((1) \& (2)). Thus the expressions (8), (9) and Equation (12) together comprise a nonlinear transformation that from the solution of Equation (12) to the solution of Equations ((1) \& (2)). According to the nonlinear transformation composed of expressions (8), (9) and Equation (12), in order to obtain the solution of Equations ((1) \& (2)), we have to give the solution of Equation (12). Many solutions of Equation (12) are easily given by

$$
\begin{gathered}
\phi_{1}=1+\mathrm{e}^{k x \mp \alpha k^{2} t}, \\
\phi_{2}=1 \mp \alpha x t+\frac{1}{6} x^{3}, \\
\phi_{3}=1+\mathrm{e}^{\mp \alpha t}(A \cosh x+B \sinh x), \\
\phi_{4}=1+\mathrm{e}^{ \pm \alpha t}(A \cos x+B \sin x),
\end{gathered}
$$

$A$ and $B$ are constants, $\cdots$, and so on.

\section{Various Exact Solutions of Equations ((1) \& (2))}

Substituting the solution $\phi_{1}=1+\mathrm{e}^{k x \mp \alpha k^{2} t}$, of Equtions (12) into (8), (9), we have the solitary wave solutions of Equations ((1) \& (2)):

$$
\begin{gathered}
u(x, t)= \pm \frac{1}{2} \alpha k\left\{1+\tanh \left[\frac{1}{2}\left(k x \mp \alpha k^{2} t\right)\right]\right\}, \\
v(x, t)=-\frac{1}{4} \alpha k^{2} \operatorname{sech}^{2}\left[\frac{1}{2}\left(k x \mp \alpha k^{2} t\right)\right] .
\end{gathered}
$$

Substituting the solution $\phi_{2}=1 \mp \alpha x t+\frac{1}{6} x^{3}$, of Equation (12) into (8), (9), we have rational solutions of Equations ((1) \& (2)):

$$
\begin{gathered}
u(x, t)=\frac{ \pm \frac{\alpha}{2} x^{2}-\alpha t}{1 \mp \alpha x t+\frac{1}{6} x^{3}}, \\
v(x, t)=-\frac{\alpha x}{1 \mp \alpha x t+\frac{1}{6} x^{3}}+\alpha \frac{\left(\frac{1}{2} x^{2} \mp \alpha t\right)^{2}}{\left(1 \mp \alpha x t+\frac{1}{6} x^{3}\right)^{2}} .
\end{gathered}
$$

Substituting the solution $\phi_{3}=1+\mathrm{e}^{\mp \alpha t}(A \cosh x+B \sinh x)$, of Equation (12) into (8), (9), we have the exact solutions containing hyperbolic functions of Equations ((1) \& (2)):

$$
\begin{gathered}
u(x, t)= \pm \alpha \frac{\mathrm{e}^{\mp \alpha t}(A \sinh x+B \cosh x)}{1+\mathrm{e}^{\mp \alpha t}(A \cosh x+B \sinh x)}, \\
v(x, t)=\frac{-\alpha \mathrm{e}^{\mp \alpha t}(A \cosh x+B \sinh x)}{1+\mathrm{e}^{\mp \alpha t}(A \cosh x+B \sinh x)}+\frac{\alpha \mathrm{e}^{\mp 2 \alpha t}(A \sinh x+B \cosh x)^{2}}{\left[1+\mathrm{e}^{\mp \alpha t}(A \cosh x+B \sinh x)\right]^{2}} .
\end{gathered}
$$

Substituting the solution $\phi_{4}=1+\mathrm{e}^{ \pm \alpha t}(A \cos x+B \sin x)$, of Equation (12) 
into (8), (9), we have the exact solutions containing trigonometric functions of Equations ((1) \& (2)):

$$
\begin{gathered}
u(x, t)= \pm \alpha \mathrm{e}^{ \pm \alpha t} \frac{(-A \sin x+B \cos x)}{1+\mathrm{e}^{ \pm \alpha t}(A \cos x+B \sin x)}, \\
v(x, t)=\frac{\alpha \mathrm{e}^{ \pm \alpha t}(A \cos x+B \sin x)}{1+\mathrm{e}^{ \pm \alpha t}(A \cos x+B \sin x)}+\frac{\alpha \mathrm{e}^{ \pm 2 \alpha t}(-A \sin x+B \cos x)^{2}}{\left[1+\mathrm{e}^{ \pm \alpha t}(A \cos x+B \sin x)\right]^{2}}
\end{gathered}
$$

$\cdots$, and so on.

It is well known that the linear Equation (12) can admit an infinite many solutions, based on the nonlinear transformation composed of (8), (9) and (12), we can obtain more solutions of Equations ((1) \& (2)), provided that more solutions of Equation (12) are given.

\section{Conclusion}

In this paper, the nonlinear transformation composed of (8), (9) and (12) for the Boussinesq-Burgers equations has been derived by using the simplified homogeneous balance method. The important role of the nonlinear transformation is that the problem of solving nonlinear Boussinesq-Burgers equations becomes that of solving a linear equation, and the latter is much easily to solve for the researchers.

\section{Acknowledgements}

The authors are very grateful to the referees for their valuable comments. The project supported in part by the National Natural Science Foundation of China (Grant Nos. 11301153, 11601225) and The Doctoral Foundation of Henan University of Science and Technology (Grant No. 09001562) and The Science and Technology Innovation Platform of Henan University of Science and Technology (Grant No. 2015XPT001).

\section{References}

[1] Li, X.M. and Chen, A.H. (2005) Darboux Transformation and Multi-Solton Solutions of Boussinesq-Burgers Equation. Physics Letters A, 342, 413-420. https://doi.org/10.1016/j.physleta.2005.05.083

[2] Khalfallah, M. (2009) Exact Travelling Wave Solutions of Boussinesq-Burgers Equation. Mathematical and Computer Modelling, 49, 666-671. https://doi.org/10.1016/j.mcm.2008.08.004

[3] Rady, A.S.A. and Khalfallah, M. (2010) On Soliton Solutions for Boussibesq-Burgers Equations. Communication in Nonlinear Science and Numerical Simulation, 15, 886-894. https://doi.org/10.1016/j.cnsns.2009.05.039

[4] Wang, P., Tian, B., Liu, W.J., Lu, X. and Jiang, Y. (2011) Backlund Transformation and Multi-Soliton Solutions for the Boussinesq-Burgers Equations from Shallow Water Waves. Applied Mathematics and Computation, 218, 1726-1734. https://doi.org/10.1016/j.amc.2011.06.053

[5] Ravi, L.K., Ray, S.S. and Sahoo, S. (2017) New Exact Solutions of Coupled Boussinesq-Burgers Equations by Exp-Function Method. Journal of Ocean Engineering and Science, 2, 34-36. https://doi.org/10.1016/j.joes.2016.09.001 
[6] Li, Q., Xia, T.C. and Chen, D.Y. (2017) 2N+1-Soliton Solutions of Boussinesq-Burgers Equation. Communications in Mathematical Research, 33, 26-32. https://doi.org/10.13447/j.1674-5647.2017.01.04

[7] Wang, M.L., Li, X.Z. and Zhang, J.L. (2014) Simplified Homogeneous Balance Method and Its Application to Whitham-Broer-Kaup Equations. Journal of Applied Mathematics and Physics, 2, 823-827. https://doi.org/10.4236/jamp.2014.28091

[8] Wang, M.L., Zhang, J.L. and Li, X.Z. (2016) Decay Mode Solutions to Cylindrical KP Equation. Applied Mathematics Letters, 62, 29-34. https://doi.org/10.1016/j.aml.2016.06.012

[9] Wang, M.L., Zhang, J.L. and Li, X.Z. (2017) N-Dimensional Auto-Bäcklund Transformation and Exact Solutions to n-Dimensional Burgers System. Applied Mathematics Letters, 63, 46-52. https://doi.org/10.1016/j.aml.2016.07.019

Submit or recommend next manuscript to SCIRP and we will provide best service for you:

Accepting pre-submission inquiries through Email, Facebook, LinkedIn, Twitter, etc. A wide selection of journals (inclusive of 9 subjects, more than 200 journals)

Providing 24-hour high-quality service

User-friendly online submission system

Fair and swift peer-review system

Efficient typesetting and proofreading procedure

Display of the result of downloads and visits, as well as the number of cited articles Maximum dissemination of your research work

Submit your manuscript at: http://papersubmission.scirp.org/

Or contact jamp@scirp.org 